Elsevier Editorial System(tm) for

Biochemical and Biophysical Research Communications

Manuscript Draft

Manuscript Number:

Title: Spatiotemporal control of mitochondrial network dynamics in astroglial cells

Article Type: SI: Mitochondrial Dynamics

Keywords: mitochondrial dynamics; astroglial cells; calcium; cell metabolism; brain injury

Corresponding Author: Dr. Matteo Bergami,

Corresponding Author's Institution: Uniklink Koeln

First Author: Jana Göbel

Order of Authors: Jana Göbel; Elisa Motori; Matteo Bergami

Abstract: Mitochondria are increasingly recognized for playing important roles in regulating the evolving metabolic state of mammalian cells. This is particularly true for nerve cells, as dysregulation of mitochondrial dynamics are invariably associated with a number of neuropathies. Accumulating evidence now reveals that changes in mitochondrial dynamics and structure may play equally important roles also in the cell biology of astroglial cells. Astroglial cells display a significant heterogeneity in their morphology and specialized functions across the different brain regions, however besides fundamental differences they seem to share a surprisingly complex meshwork of mitochondria, which is highly suggestive of tightly regulated mechanisms that contribute to maintain this unique architecture. Here, we summarize recent work performed in astrocytes in situ indicating that this may indeed be the case, with astrocytic mitochondrial networks shown to experience rapid dynamic changes in response to defined external cues. Although the mechanisms underlying this degree of mitochondrial re-shaping are far from being understood, recent data suggest that they may contribute to demarcate astrocyte territories undergoing key signalling and metabolic functions. 


\section{Spatiotemporal control of mitochondrial network dynamics in astroglial cells}

Jana Göbel $^{1}$, Elisa Motori ${ }^{2}$ and Matteo Bergami ${ }^{1,3}$

${ }^{1}$ Cologne Excellence Cluster on Cellular Stress Responses in Aging-Associated Diseases (CECAD) and University Hospital of Cologne, Joseph-Stelzmann-Straße 26, D-50931 Cologne, Germany.

${ }^{2}$ Department of Mitochondrial Biology, Max Planck Institute for Biology of Ageing, JosephStelzmann-Straße 9B, D-50931 Cologne, Germany.

${ }^{3}$ Center for Molecular Medicine (CMMC), University of Cologne, Robert-Koch-Str. 21, D-50931 Cologne, Germany

Key words: mitochondrial dynamics; astroglial cells; calcium; cell metabolism; brain injury

Correspondence to: Matteo Bergami

CECAD-University Hospital of Cologne, Germany

Email: matteo.bergami@uk-koeln.de

Phone: +49 (0)221 47884250 


\section{Highlights}

- Astrocytes display highly complex mitochondrial architectures in vivo

- Mitochondrial trafficking and dynamics in astrocytes are regulated

- Mitochondria critically contribute to astrocyte $\mathrm{Ca}^{2+}$ handling

- Astrocyte reacting to injury undergo marked changes in mitochondrial dynamics 


\begin{abstract}
Mitochondria are increasingly recognized for playing important roles in regulating the evolving metabolic state of mammalian cells. This is particularly true for nerve cells, as dysregulation of mitochondrial dynamics are invariably associated with a number of neuropathies. Accumulating evidence now reveals that changes in mitochondrial dynamics and structure may play equally important roles also in the cell biology of astroglial cells. Astroglial cells display a significant heterogeneity in their morphology and specialized functions across the different brain regions, however besides fundamental differences they seem to share a surprisingly complex meshwork of mitochondria, which is highly suggestive of tightly regulated mechanisms that contribute to maintain this unique architecture. Here, we summarize recent work performed in astrocytes in situ indicating that this may indeed be the case, with astrocytic mitochondrial networks shown to experience rapid dynamic changes in response to defined external cues. Although the mechanisms underlying this degree of mitochondrial re-shaping are far from being understood, recent data suggest that they may contribute to demarcate astrocyte territories undergoing key signalling and metabolic functions.
\end{abstract}




\section{TEXT}

Astrocytes are an abundant cell population that fulfils a range of functions, from regulating neurovascular coupling to local refinement in synapse structure and function[1,2,3]. The multifunctional role of these cells is mirrored morphologically by a highly complex yet welldelineated arrangement of branches and branchlets. On the one hand, these exceedingly thin processes provide a dynamic coverage of neuronal synaptic contacts, thereby regulating their formation, maturation and ultimately maintenance[1]. On the other, elaborated perivascular processes almost completely sheath the brain microvasculature through highly specialized terminals called end-feet, proposed to co-regulate vascular tone as well as blood-brain-barrier function[4,5]. Accumulating evidence indicates that most of the specialized signaling functions that perisynaptic and perivascular astrocytic processes fulfill are invariably mediated by local, regionalized changes in second messengers (e.g. $\mathrm{Ca} 2+$ ) associated with the secretion of transmitters and other metabolites[3,6,7], thereby suggesting the existence of well-organized compartments that timely regulate the availability of these molecules in concert with, e.g. neuronal activity or vascular/interstitial flow. One organelle likely involved in this regulation is the mitochondrial network, which can effectively couple alternating periods of high/low respiration with local changes in ions availability and metabolite supply[8,9,10,11], thereby potentially coordinating important intracellular signaling events in astrocytes. Interestingly, from an energy metabolism point of view it is well established that astrocytes have prevalent glycolytic and glycogenic rates whereas the precise extent of ongoing oxidative phosphorylation (OXPHOS) in vivo has remained a matter of debate for long time. By sorting astrocytes from whole mouse brains, several recent studies have now started to provide evidence that, alongside with a high expression of glycolytic enzymes, these cells are also enriched in several enzymes of the tricarboxylic acid (TCA) cycle[12,13], thus not only supporting the notion that astrocytes have a prominent oxidative capacity but also emphasizing their ability to flexibly modify their metabolic profile according to substrate availability and/or energy demand[10,14]. This degree of metabolic "plasticity" may also reflect the peculiar capability 
of astrocytes to adapt to extreme conditions of metabolic challenge, as for instance during ischemia, injury and inflammation. This has been exemplified, for example, in cultured astrocytes that preferentially increase their rate of glycolysis at the expenses of mitochondrial respiration to maintain ATP production when directly exposed to pro-inflammatory stimuli[15,16,17]. Intriguingly, this seemingly unique ability to rapidly adapt their cellular energy state appears to reflect the pronounced changes in morphology, gene and marker expression that mark the acquisition of astrocytic reactive states during disease[18]. Thus, although the precise cellular and sub-cellular events underlying the progression through these evolving states of reactivity are only partly understood, changes in mitochondrial structure and function are becoming important hallmarks of the astrocytic response to brain insults. Here we present some of the most recent observations revealing the peculiar nature of the mitochondrial network in astrocytes, and discuss how dynamic changes in its architecture may contribute to regulate fundamental functions in astrocytes both under resting conditions and in response to injury.

\section{Unique architecture of the astrocytic mitochondrial network}

Owing to a very complex tridimensional morphology, which in most astrocytes is characterized by a number of major processes giving rise to thousands of ramified branchlets and leaflets, a detailed analysis of the mitochondrial network architecture in these cells in situ has been elusive. Only during the last decade electron microscopy studies have started to ascertain that astrocytes, and astroglial cells in general, are surprisingly enriched in mitochondria[12,19,20,21]. These organelles have been found invading most of the peripheral processes, including fine branchlets and terminal endfeet, where highly heterogeneous organelle morphologies were observed, presumably suggesting the capacity of the mitochondrial network to accommodate a certain degree of structural remodeling to match the spatial constrains of perineuronal and perivascular processes. In particular, within endfeet processes large bundles of both small and elongated, branched and unbranched mitochondria were described in close apposition to the perivascular membrane[19]. At the 
perisynaptic level, however, the physical size of some of the finest astrocytic processes (so-called PAPs, tens of nanometers wide) appears to essentially prevent the entry of cellular organelles, yet mitochondria are often observed in the immediate apposition to these fine terminals[11,22,23,24]. Similar observations suggesting the existence of a highly complex mitochondrial network in protoplasmic astrocytes in vivo were independently provided by few recent studies utilizing high resolution light microscopy and genetically-encoded fluorescent reporters (mito-GFP or similar, either delivered by a virus-based approach[16] or expressed by inducible reporter mice[25,26]), to specifically illuminate astrocytic mitochondria. These works describe the existence of a densely packed network of highly interconnected mitochondria occupying a substantial volume of individual astrocytes, particularly main processes and branches, but also reaching fine protrusions and endfeet[16,26]. The morphology of these organelles in astrocytes was also confirmed to be rather heterogeneous, yet while a dense meshwork of elongated mitochondria was found in the soma and within main branches, thinner and shorter mitochondria mostly populated peripheral processes, including perineuronal protrusions. With slight variability, this peculiar distribution appears to be maintained across the different layers of the cortex (Figure 1A), yet analysis of mitochondrial network architecture in astroglia of other brain regions including striatum, hippocampus and cerebellum disclosed a certain degree of differences (Figure 1B), on account of essentially distinct astrocytic morphologies. These features therefore indicate that fundamentally distinct sub-populations of astrocytes across the brain may display an equally different architecture of their mitochondrial network, suggesting that these organelles may efficiently adapt to unique morphological constrains imposed by region-specific astrocyte geometries. It is also tempting to speculate that some of the anatomical differences observed in the diverse brain areas may reflect a broader reorganization of astrocytic mitochondrial networks owing to metabolic adaptations to activity patterns of, e.g., specific neuronal circuits or even subtypes of nearby synapses. Interestingly, the observed heterogeneity in astrocytic mitochondrial networks may mirror the increasingly recognized morphological and functional diversity of these cells, which is likely 
determined by a combination of both intrinsically encoded factors and external cues provided by the surrounding microenvironment[27].

\section{Mitochondrial trafficking and dynamics in astrocytes in situ are regulated}

The complex and seemingly heterogeneous structure of the mitochondrial network in astrocytes is highly suggestive of prominent ongoing trafficking and dynamics of mitochondria. In mammals, experiments conducted predominately in neurons revealed that specific adaptor proteins (e.g., Miro and TRAKs) sit right at the core of the mitochondrial trafficking machinery, and mediate the reversible binding of mitochondria to proper motor proteins (kinesin and dynein)[28]. While the exact stoichiometry of these core elements are still debated, at least some of the mechanisms linking mitochondrial transport to synapse function have been proposed (e.g. via $\mathrm{Ca}^{2+}$-sensing domains of the adaptor proteins, see below), as well as their implications in several neurological disorders[29]. On the other side, the proteins regulating mitochondrial dynamics (namely, fusion and fission) and how their dysfunction may lead to secondary alterations in mitochondrial structure and respiratory capacity, are a matter of equally intense investigation due to their direct association to human diseases. Indeed, pathogenic mutations in some of these proteins have been directly linked with the onset of certain classes of hereditary neuropathies, e.g. Charcot-Marie-Tooth disease type 2A[30] and dominant optic atrophy[31,32], as well as to abnormal brain development[33]. The chief mediators of mitochondrial dynamics include several dynamin-related GTP-binding proteins located on the outer and inner mitochondrial membranes, where they promote the catalytic reactions leading to fusion or division of mitochondria[34,35]. Fusion of the outer membrane is governed by mitofusin 1 and 2, while the protein optic atrophy 1 (OPA1) and in particular the balanced proteolytic processing of its several splice variants into long and short isoforms mediates inner mitochondrial fusion/fission dynamics[36]. In contrast, mitochondrial fission at the outer mitochondrial membrane is promoted by the dynamin-related protein 1 (Drp1), a cytosolic protein whose re-localization to/from the membrane is regulated by its phosphorylation state on distinct 
serine residues, as well as by other post-transcriptional modifications (i.e., ubiquitylation, Snitrosylation and SUMOylation)[37]. While the recruitment and subsequent assembly of Drp1 at the precise sites of mitochondrial fission is facilitated by other membrane adaptor proteins (e.g., mitochondrial fission factor, MFF, and mitochondrial fission protein, FIS1)[34,37], recent evidence suggests that the choice of these fission sites may be pre-determined by a local, dynamic rearrangement of nearby ER tubules who first mark and then physically constrict the mitochondrial membrane to allow Drp1 assembly[38]. Maintenance of an efficient mitochondrial network thus seems to be regulated by a complex choreography of membrane contact sites, fission and fusion events, whereby manipulating the expression of any of their regulatory proteins easily destabilizes this delicate balance by producing either a highly fragmented or hyper-tubulated network. Evidence in vitro indicates that astrocytes display substantial rates of mitochondrial fusion[39,40] in line with their overtly dynamic and motile network (Figure 2A-B). Further evidence also indicates that astrocytes respond to at least some of the aforementioned gene expression manipulations of dynamin-related proteins by rapidly altering their mitochondrial network morphology[16,41], although the precise consequences of these manipulations in astroglial cells have not been tested systematically yet. Nonetheless, it is reasonably safe to assume that, in glial cells as in other cell types, mitochondrial dynamics may be of key relevance for preserving quality control mechanisms and the intermixing of mitochondrial content (e.g., mtDNA and proteins), and thus facilitate changes in respiratory capacity to meet the evolving cellular demands[35]. It is also important to consider that as for many other cell types, most of these studies have assessed the extent and regulation of astrocytic mitochondrial dynamics in culture, where the phenotypic characteristics of these otherwise highly differentiated cells become significantly altered in terms of morphology and gene expression. For instance, astrocytes in culture lose their tri-dimensional polarized morphology by acquiring a planar geometry, also reflecting the lack of perisynaptic and -vascular processes. Moreover, astrocytes in culture are proliferative, in marked contrast to post-weaning/adult brain tissue, and their gene expression profile is more similar to a de-differentiated progenitor- or 
reactive-like state[42]. Interestingly, the advent of accessible confocal and 2-photon microscopy techniques alongside with gene manipulation technology allowed starting to address how astrocyte mitochondrial dynamics and trafficking may be regulated in situ in mouse brain explants. Recent imaging work in brain slices following expression of mitochondrially-targeted fluorophores (GFP or similar) revealed that also in these settings, astrocytes possess a certain degree of mitochondrial movement (quantified as displacement), albeit at a level consistently lower than previously observed in culture. For instance, fluorescent labelling of the mitochondrial network via biolistic transfection[43] or viral transduction[44] of astrocytes in cultured organotypic brain slices revealed a predominant proportion of stationary organelles over the course of several minutes of imaging, with only $20-30 \%$ of them displaying active movement. Along the same vein, analysis of individual mitoGFP-expressing astrocytes in freshly cut, acute brain slices shows that the extent of mitochondrial displacement remains rather stable at a value of about $20 \%$ over the course of up to 2 hours of imaging (Figure 2C-G). While the measurements in these experiments cannot fully discriminate between net trafficking vs. fission/fusion or other remodeling dynamics of mitochondria, they provide a first estimate of their rate of overall temporal "displacement" in living cells within their native tissue. Interestingly, by focusing on peripheral astrocytic processes where isolated mitochondria could be better discerned from the rest of the network, recent works have begun to more specifically investigate the relative trafficking of these organelles in astrocytes, utilizing kymograph analysis similar to what has been previously done for neuronal axonal processes[45]. These studies show that astrocytic mitochondrial trafficking is consistently affected by slice manipulation of neuronal activity, with blockers (e.g., TTX) increasing whereas enhancers (glutamate or electric stimulation) reducing mitochondrial motility[43,44]. While this sort of activity-dependent repositioning of astrocytic mitochondria appeared to correlate with the spatial distribution of synapses of nearby neurons in slices, it is noteworthy to mention that also mitochondrial fusion dynamics - as evaluated with a photo-activatable mitochondrial probe - were sensitive to glutamate application, who produced a decrease in the basal rate of fusion[44]. 
Therefore, changes in both trafficking and fusion dynamics of mitochondria in cultured brain tissue explants may be modulated by the application of neurotransmitters, suggesting that astrocytes may reorganize their mitochondrial network in register with changes in network excitability. Whether this local reorganization of the mitochondrial network may in turn (directly or indirectly) modulate synaptic efficacy, for instance by contributing to local $\mathrm{Ca}^{2+}$ homeostasis in perisynaptic processes or by regulating the availability of transmitters, is unknown. Thus, future studies will have to address the question whether manipulations specifically perturbing either mitochondrial repositioning or fusion dynamics in astrocytes may produce any measurable effects on nearby synapses and, more broadly, on circuit functioning in a physiological context.

\section{Mutual regulation of mitochondrial and $\mathrm{Ca}^{2+}$ dynamics in astrocytes}

On account of their highly ramified morphology as well as expression of a wide repertoire of membrane receptors, transporters and channels, astrocytes are thought of regulating locally tissue homeostasis, including ions concentration and neuro/glio-transmitter uptake and release[3]. At the core of this capability of sensing and signaling regionalized changes in the activity of, e.g., nearby synapses is a very unique spatiotemporal regulation of $\mathrm{Ca}^{2+}$ events[46,47]. In fact, unlike neurons, astrocytes are electrically silent, however they display a surprisingly intense activity in $\mathrm{Ca}^{2+}$ dynamics, posing the question which intracellular stores may contribute to precisely shape these complex patterns of $\mathrm{Ca}^{2+}$ signaling events. Interestingly, $\mathrm{Ca}^{2+}$ transients in astrocytes seem to occur spontaneously, but they can also be triggered by specific stimulation with neurotransmitters and, in vivo, by sensory stimuli and locomotor activity[48,49]. Accumulating evidence from ex vivo and in vivo high-resolution confocal and 2-photon imaging studies suggests that astrocyte display a remarkable variety of $\mathrm{Ca}^{2+}$ dynamics, which may help explaining how $\mathrm{Ca}^{2+}$ signaling events in these cells can differentially transduce a diversity of stimuli and generate, in turn, specific effects at synapses and perivascular endfeet[47,50]. For instance, a large fraction of the $\mathrm{Ca}^{2+}$ events an astrocyte experiences during a typical imaging session of several minutes is essentially 
characterized by transients extremely localized (usually spanning few $\mu \mathrm{m}$ ), asynchronous in nature and of somewhat variable duration (from very fast to longer-lasting "expanding" transients), in contrast to less frequent but pronounced raises occurring in the cell soma[51]. On the one side, the temporal scale of these localized, fast events can reconcile the presumptive role of perisynaptic astrocytic processes in modulating synaptic function[3]. On the other, the identification of these functionally independent micro- (few $\mu \mathrm{m}$ in size) and expanding $\mathrm{Ca}^{2+}$ domains (also referred as to "waves", usually covering an area larger than microdomains) within astrocytic processes, together with their varied kinetics, has led to the hypothesis that their occurrence may facilitate the temporal “integration" of extracellular signals[47], thus strongly suggesting that astrocytes might effectively decode and thus respond to specific patterns of neuronal activity[3]. It is therefore not surprising that substantial effort has been made during the last few years in trying to understand what mechanisms regulate $\mathrm{Ca}^{2+}$ homeostasis and microscopic compartmentalization of signaling events in astrocytes[52,53,54,55,56]. Interestingly, the first obvious candidate and major intracellular pathway of $\mathrm{Ca}^{2+}$ homeostasis, i.e. the inositol triphosphate (IP3)-dependent release from the ER stores, appears to contribute mainly to large somatic $\mathrm{Ca}^{2+}$ events, having only partial effects on the generation and frequency of fluctuations occurring in the astrocytes' processes[54,57]. By contrast, an appreciable amount of local $\mathrm{Ca}^{2+}$ events, as detected by utilizing membrane-targeted geneticallyencoded calcium indicators, rather relies upon distinct sources, and amongst these, mitochondria have recently emerged as key candidates. Indeed, in a recent study performed in astrocytes in situ, mitochondria were not only often found sitting in very close proximity to recorded microdomains but $\mathrm{Ca}^{2+}$ efflux in particular through the mitochondrial permeability transition pore (mPTP), a nonselective channel located at the inner mitochondrial membrane, was shown to directly supply these local signaling events[26]. As the authors of this study suggest, these observations may highlight the presumable capability of astrocytic mitochondria to dynamically couple periods of high respiration with the simultaneous generation of discrete $\mathrm{Ca}^{2+}$ signaling domains[26]. Indeed, $\mathrm{Ca}^{2+}$ efflux through periodic opening of the mPTP has been postulated as a mechanism capable of 
ensuring the dissipation of excessive intra-mitochondrial $\mathrm{Ca}^{2+}$ accumulation (thus preventing mitochondrial overload and damage) in regions of the cells anyhow subjected to elevated $\mathrm{Ca}^{2+}$ fluxes (i.e., IP3R- or transmembrane-mediated)[58]. In this case, transient mitochondrial $\mathrm{Ca}^{2+}$ efflux may concurrently contribute to amplify spatially-restricted signaling events. It is established that mitochondrial $\mathrm{Ca}^{2+}$ uptake occurs via the low-affinity $\mathrm{Ca}^{2+}$ uniporter (MCU), whose opening is modulated by several accessory and regulatory proteins[8]. Interestingly, a number of studies performed in different cell types suggest that under physiological conditions, mitochondrial $\mathrm{Ca}^{2+}$ uptake occurs at spatially restricted intracellular locations subjected to particularly elevated $\mathrm{Ca}^{2+}$ concentrations[8], as those generated in close proximity ( 30-100 $\mathrm{nm})$ [59] to IP3 receptors on the ER (i.e., so-called mitochondria-ER tethering domains)[60], or to $\mathrm{Ca}^{2+}$ channels on the plasma membrane. Mitochondrial $\mathrm{Ca}^{2+}$ accumulation through $\mathrm{MCU}$ complex is then counteracted by the activity of the $\mathrm{Na}^{+}$and $\mathrm{H}^{+} / \mathrm{Ca}^{2+}$ antiporters located on the inner mitochondrial membrane or possibly even through transient mPTP opening, which altogether modulate the extent and spread of cytosolic $\mathrm{Ca}^{2+}$ transients[58,61]. To some extent, this has been also assessed in astrocytes in culture, for instance utilizing mitochondrially-targeted genetic indicators to monitor $\mathrm{Ca}^{2+}$ influx/efflux from these organelles[62,63]. The fact that mitochondria have been now directly suggested as key regulators of the "de novo" generation of fast $\mathrm{Ca}^{2+}$ events in astrocyte processes is therefore surprising, as not only the exceptionally rapid kinetics of these events would appear in contradiction with the somewhat much slower $\mathrm{Ca}^{2+}$ efflux from mitochondria[63], but to take effect this mechanism would also benefit from a long-lasting $\mathrm{Ca}^{2+}$ storage capacity in astrocytic mitochondria presumably independent from other surrounding $\mathrm{Ca}^{2+}$ sources. In support of this newly-proposed mechanism, experiments conducted following depletion of ER stores via thapsigargin or the use of IP3R2 knock-out astrocytes (in which IP3-dependent $\mathrm{Ca}^{2+}$ release from the ER is prevented) revealed that the persisting amount of fast $\mathrm{Ca}^{2+}$ events detected was sensitive to pharmacological inhibition of mitochondrial $\mathrm{Ca}^{2+}$ efflux[26]. However, experiments conducted in wild-type astrocytes of acute slices perfused with $\mathrm{Ca}^{2+}$ free media also demonstrated that these fast events are 
primarily dependent on extracellular $\mathrm{Ca}^{2+}$ influx[57], suggesting the possibility that rather than generating $\mathrm{Ca}^{2+}$ microdomains, mitochondria may contribute to confine their magnitude and spread in proximity of the plasma membrane. Conceivably, in order to efficiently and rapidly influence localized changes in astrocytic $\mathrm{Ca}^{2+}$ homeostasis, this mitochondrial-dependent mechanism would require the existence of a widespread mitochondrial meshwork invading the most peripheral processes, which is indeed the type of structure observed in astrocytes (see also paragraph above). Yet, the presumable dynamic nature of the mitochondrial network in astrocytes, together with the well-described spatial constrains of the $\mathrm{Ca}^{2+}$ microdomains, would necessitate of mechanisms guiding the precise locations where mitochondria are most needed to exert these functions, and thus an efficient machinery regulating fusion-fission, membrane tethering as well as repositioning of mitochondria would be beneficial to let these organelles not only access but also demarcate sites of higher metabolic demand[64]. Interestingly, work performed ex vivo started to address, at least in part, what these mechanisms might be. Not entirely unexpected, motor adaptor proteins such as Miro1 and 2 have been proposed to drive also in astrocytes, as in neurons[45,65], the repositioning of mitochondria in a $\mathrm{Ca}^{2+}$-dependent manner along the cells' processes. Miro1 and 2 are atypical Rho-GTPase proteins equipped with two $\mathrm{Ca}^{2+}$-sensing EF-hands and a C-terminal transmembrane domain that mediates their localization to the mitochondrial outer membrane. Through conformational changes induced by $\mathrm{Ca}^{2+}$ binding, these proteins can reversibly associate with kinesin and dynein motors to promote mitochondrial trafficking along microtubules. Although the exact mechanisms regulating these conformational changes are still debated[29], experiments conducted in astrocytes in organotypic slices show that Miro proteins regulate the movement of mitochondria both under basal conditions and following induced stimulation in a $\mathrm{Ca}^{2+}$-dependent manner[44,66]. While mitochondria thus become immobilized at sites of higher $\mathrm{Ca}^{2+}$ concentrations, disruption of Miro activity in astrocytes not only impairs the "activity-dependent" motility of their mitochondria, but also seems to influence the occurrence of cytosolic $\mathrm{Ca}^{2+}$ signaling domains[44,66]. If proven in a more physiological context, i.e. in preparations in vivo, the 
fact that Miro proteins may regulate in a calcium-dependent manner mitochondria positioning in astrocyte processes poses the interesting question which primary and secondary mechanisms may mutually orchestrate $\mathrm{Ca}^{2+}$ homeostasis at spots of intense activity. Yet, the complex structure of the astrocytic mitochondrial network in vivo might suggest that the actual extent of net mitochondrial trafficking might not be as pronounced as observed in preparations ex vivo or in vitro. Mature protoplasmic astrocytes are indeed densely packed with mitochondria, with only very few of the optically resolved, peripheral fine processes left unoccupied[16,26]. What is more, mitochondria are continuously exposed to an intense variety of $\mathrm{Ca}^{2+}$ events throughout the astrocytes' volume, even in basal conditions, as mentioned above. It therefore seems unlikely that these organelles may undergo extensive or very frequent movements along astrocytic processes, in contrast to the much longer axonal processes where overt trafficking has been documented[67], thus suggesting that Miro proteins may preferentially contribute to co-regulate $\mathrm{Ca}^{2+}$ buffering capacity independent of their role in mitochondrial transport[68], at least in fully differentiated, adult astrocytes. On the contrary, during early post-natal stages when astrocytes are still maturing and may migrate or proliferate to populate their final locations in the brain[69], or even in a setting of injury in which astrocytes enter a state of reactivity marked by pronounced metabolic changes[42], Miro proteins may effectively play a significant role in regulating the positioning of mitochondria to match the evolving spatial metabolic needs of the cell. It is also not clear to which extent mitochondrial fusion-fission dynamics contribute to regulate, in astrocytes in situ, homeostatic changes in $\mathrm{Ca}^{2+}$ buffering at cellular microdomains. Growing evidence in astrocyte cultures suggests that this might be the case, as changes in mitochondrial dynamics and remodeling have been linked to $\mathrm{Ca}^{2+}$ handling, although these studies point to a primary dependence upon mitochondrial membrane potential and ROS production as the leading events involved in homeostatic $\mathrm{Ca}^{2+}$ changes[70,71,72,73,74]. Nonetheless, given the proposed role of at least one protein, i.e. Mfn2, in regulating the extent of mitochondria-ER functional tethering besides mitochondrial fusion in cells[75], it is tempting to speculate a direct involvement of mitochondrial dynamics in maintaining 
the heterogeneity in $\mathrm{Ca}^{2+}$ signaling domains observed in astrocyte processes[47]. With the concurrent optimization of advanced genetically-encoded indicators and imaging technologies[76,77], future studies will therefore be needed not only to better clarify the actual role of $\mathrm{Ca}^{2+}$-sensing, mitochondrial trafficking proteins in astrocytes in vivo, but also the precise mutual regulation of $\mathrm{Ca}^{2+}$ homeostasis/signaling and mitochondrial dynamics in these highly differentiated cells.

\section{Mitochondrial dynamics and quality control in astrocytes reacting to injury}

Over the course of the last years, astrocytes have gradually but progressively emerged as a group of cells invariably involved in virtually most brain diseases. While current evidence indicates that astrocytes do exert various and important functions in the healthy brain, presumably owning to their increasingly recognized cellular heterogeneity[27], to date we still know surprisingly little about the sub-cellular changes these cells experience when facing challenging and harmful conditions. Astrocytes are well recognized for their capability to markedly up-regulate classic markers of "gliosis" in response to tissue damage, degeneration and inflammation, however they have been also recently re-evaluated in terms of structural and functional changes, including gene expression profile, depending on the type of injury and anatomical location[18]. For instance, several lines of evidence now indicate that astrocytes can significantly adapt their metabolic state to distinct insults, thus revealing a previously unknown layer of complexity in the processes regulating the acquisition of reactive states in these cells[42,78]. While this form of plasticity is likely to involve multiple intracellular pathways and organelles, recent data suggest that the evolving state of reactive astrocytes is mirrored by a corresponding alterations of their mitochondrial network, the central player in energy metabolism and metabolic signalling. For instance, overall changes in mitochondrial markers and ultrastructure have been commonly described in several brain diseases or following traumatic injury/stroke in rodent models as well as human post-mortem material[79,80,81,82,83,84]. Also, marked changes in mitochondrial membrane potential, redox 
state and $\mathrm{Ca}^{2+}$ influx have been linked in astrocytes, as in neurons, to several types of injury and excitotoxicity[80,85] via mechanisms likely involving the opening of the MPTP, which as mentioned earlier may lead to rapid dissipation of membrane potential and uncoupling of OXPHOS. Interestingly, accumulating evidence suggests that these are also common events occurring during brain trauma and ischemia, as well as in several neurodegenerative disorders, yet most of these data originate from in vitro or post-mortem studies, thus lacking sufficient molecular understanding to separate cause from consequence in the disease, in particular in the case of astrocytes. Likewise, neuropathologies that accompany proper mitochondrial diseases[86] often involve gliosis in conjunction with neuronal loss and axon degeneration, however whether alterations in astrocytic mitochondria significantly contribute to regulate the onset or progressive accumulation of irreversible tissue damage is unclear. While some studies have thus begun to utilize more sophisticated mouse models to assess the actual possible contribution of astrocytic mitochondrial function/dysfunction in the pathogenesis of brain diseases[87,88,89], the precise mechanisms underlying mitochondrial structural and functional changes during astrocyte reactivity in vivo are still poorly understood. One of the reasons for this lack of knowledge appears to be the paucity of methodologies to properly dissect the actual changes in the astrocytic mitochondrial network with sufficient spatiotemporal resolution, and assign them specific roles in influencing the metabolic state of reactive cells. While this task may conceivably require the development and optimization of new genetic tools[90], some promising results in this respect have nevertheless been provided by few recent studies. For instance, we now know that the mitochondrial network in protoplasmic astrocytes responding to cortical injury rapidly undergoes a morphological remodelling which follows the temporal progression of classic traits of reactivity (e.g., GFAP expression levels). In particular, the pro-inflammatory microenvironment resulting from the initial injury insult was shown to trigger a marked but localized reorganization of the otherwise tubular mitochondrial network in astrocytes, by promoting fragmentation and the generation of smaller mitochondria, independent of visible changes in cell viability[16]. These morphological alterations, which are 
mediated by the recruitment onto astrocytic mitochondria of the pro-fission protein Drp1, are reminiscent of what has been described in cell culture[91] or in neurons after stroke[92], yet in astrocytes this phenomenon appears to large degree reversible in nature, reflecting the onset of quality-control (and presumably other) mechanisms that may contribute to limit excessive mitochondrial damage[16]. This would be in line with the notion that mitochondrial dynamics in cells contribute to maintain overall mitochondrial functionality, on the one side by promoting mitochondrial content mixing via fusion while on the other favouring segregation and elimination of irreversibly damaged organelles via fission[93]. Thus, the fact that astrocytes respond in vivo to stressful insults by dynamically reorganizing their mitochondrial network strongly suggest that this degree of remodelling may play a pivotal role in regulating energetic and metabolic challenges at the site of injury, although the underlying operating mechanisms remain to be addressed. Interestingly, forced astrocytic expression of a mutant isoform of the mitochondrial enzyme superoxide dismutase 1 ( $\left.\mathrm{SOD} 1^{\mathrm{G} 93 \mathrm{~A}}\right)$, which has been linked to amyotrophic lateral sclerosis and induces neurotoxic phenotypes in astrocytes[94,95,96], causes mitochondrial oxidative stress with ensuing dysregulation of $\mathrm{Ca}^{2+}$ homeostasis[26]. Whether this manipulation may mimic some of the features occurring in injury-induced reactive astrocytes is not known, however it reveals a plausible mechanism how damaged mitochondria may aggravate glial cells' reactivity and, possibly, neurotoxicity[88]. While the cell-autonomous implications of these changes in mitochondrial structure and function for astroglial cell reactivity are still poorly understood, and will likely be the focus of intense future investigation, recent works already begun to put forward the hypothesis that astrocytes may be involved in the intercellular transfer of mitochondria with neurons in vivo, particularly in a setting of injury. For example, it was proposed that during stroke astrocytes could transfer sill-functional mitochondria to nearby neurons via vesicular exocytosis in order to sustain neuronal metabolic activity and recovery[97], a phenomenon which is reminiscent of what has been previously described in injured lung cells or tumor models[98]. Likewise, reverse vesicular transfer of mitochondria from retinal ganglion axons to astrocytes was also shown as a mechanism of 
transcellular mitochondrial recycling[99]. While these observations shed new light on the possible mitochondrial-dependent quality control mechanisms underlying neuroglia cross-talk, they also raise important points of criticism, some of which call for further work in order to corroborate these results[100]. For example, astrocyte-to-neuron transfer of mitochondria has been primarily detected in experiments conducted in cell cultures and utilizing soluble fluorescent dyes[97], which altogether may prevent a rigorous assessment of the specificity of this mechanism, especially in subsequent functional experiments conducted in vivo. Also, no real mechanisms were provided in these studies as to how mitochondria might be tagged and separated from the existing network for vesicular loading and transfer. Are fission-fusion dynamics and trafficking at specific perineuronal processes involved in the segregation of these mitochondria, somewhat similar to what has been proposed for cell-autonomous recycling of dysfunctional mitochondria via, e.g., autophagymediated mechanisms[93]? And are such processes specific for mitochondria or also take place for other organelles? Future studies will be needed to address these points and clarify what the mechanisms are that regulate astrocyte non- and cell-autonomous trafficking as well as disposal of mitochondria in vivo, with particular attention to how this relates to the metabolic cellular state in a setting of disease, where the functional contribution of reactive astrocytes is increasingly recognized[101,102].

\section{Conclusions}

Despite growing evidence for an important role of astrocytes in modulating synaptic and vascular functions, still little is known about how these cells may efficiently couple their response to external stimuli with local intracellular changes in their signalling and metabolic state. Our knowledge about the molecular processes underlying these changes is still rudimentary, yet recent progress in imaging techniques and genetic tools allowed to start unveiling a previously unappreciated complexity in the players regulating the type and compartmentalization of the metabolic events occurring throughout the astrocytes' territories. The particularly elaborated and dynamic structure 
of the astrocytic mitochondrial network directly implicates mitochondria as potential co-regulators in most of the signalling pathways observed to date in astrocytes. Nonetheless, a systematic analysis of the actual contribution of mitochondria to astrocyte functions in vivo is still missing. Revealing the mechanisms underlying mitochondrial remodelling in astrocytes, as well as the interplay between mitochondria and other astrocytic organelles, may enable us to better dissect the evolving metabolic state of these cells while reacting to injury and disease, and thus significantly broaden our understanding of the events regulating tissue damage and repair.

\section{Funding}

This work was supported by the UoC advanced post-doc grant program, Deutsche Forschungsgemeinschaft (CRC 1218) and European Research Council (ERC-StG-2015, grant number 67844) to M.B.

\section{Conflict of interest}

No potential conflicts of interest were disclosed.

\section{References}

[1] L.E. Clarke, B.A. Barres, Emerging roles of astrocytes in neural circuit development, Nat Rev Neurosci 14 (2013) 311-321.

[2] M. Belanger, I. Allaman, P.J. Magistretti, Brain energy metabolism: focus on astrocyte-neuron metabolic cooperation, Cell Metab 14 (2011) 724-738.

[3] A. Araque, G. Carmignoto, P.G. Haydon, et al., Gliotransmitters travel in time and space, Neuron 81 (2014) 728739.

[4] D. Attwell, A.M. Buchan, S. Charpak, et al., Glial and neuronal control of brain blood flow, Nature 468 (2010) $232-$ 243.

[5] Z. Zhao, A.R. Nelson, C. Betsholtz, et al., Establishment and Dysfunction of the Blood-Brain Barrier, Cell 163 (2015) 1064-1078.

[6] H.B. Choi, G.R. Gordon, N. Zhou, et al., Metabolic communication between astrocytes and neurons via bicarbonateresponsive soluble adenylyl cyclase, Neuron 75 (2012) 1094-1104.

[7] P. Machler, M.T. Wyss, M. Elsayed, et al., In Vivo Evidence for a Lactate Gradient from Astrocytes to Neurons, Cell Metab 23 (2016) 94-102.

[8] R. Rizzuto, D. De Stefani, A. Raffaello, et al., Mitochondria as sensors and regulators of calcium signalling, Nat Rev Mol Cell Biol 13 (2012) 566-578.

[9] M.P. Murphy, How mitochondria produce reactive oxygen species, Biochem J 417 (2009) 1-13.

[10] L.F. Barros, Metabolic signaling by lactate in the brain, Trends Neurosci 36 (2013) 396-404. 
[11] B. Pardo, T.B. Rodrigues, L. Contreras, et al., Brain glutamine synthesis requires neuronal-born aspartate as amino donor for glial glutamate formation, J Cereb Blood Flow Metab 31 (2011) 90-101.

[12] D. Lovatt, U. Sonnewald, H.S. Waagepetersen, et al., The transcriptome and metabolic gene signature of protoplasmic astrocytes in the adult murine cortex, J Neurosci 27 (2007) 12255-12266.

[13] J.D. Cahoy, B. Emery, A. Kaushal, et al., A transcriptome database for astrocytes, neurons, and oligodendrocytes: a new resource for understanding brain development and function, J Neurosci 28 (2008) 264-278.

[14] L. Hertz, L. Peng, G.A. Dienel, Energy metabolism in astrocytes: high rate of oxidative metabolism and spatiotemporal dependence on glycolysis/glycogenolysis, J Cereb Blood Flow Metab 27 (2007) 219-249.

[15] A. Almeida, J. Almeida, J.P. Bolanos, et al., Different responses of astrocytes and neurons to nitric oxide: the role of glycolytically generated ATP in astrocyte protection, Proc Natl Acad Sci U S A 98 (2001) 15294-15299.

[16] E. Motori, J. Puyal, N. Toni, et al., Inflammation-induced alteration of astrocyte mitochondrial dynamics requires autophagy for mitochondrial network maintenance, Cell Metab 18 (2013) 844-859.

[17] M. Belanger, I. Allaman, P.J. Magistretti, Differential effects of pro- and anti-inflammatory cytokines alone or in combinations on the metabolic profile of astrocytes, J Neurochem 116 (2011) 564-576.

[18] M.V. Sofroniew, Astrogliosis, Cold Spring Harb Perspect Biol 7 (2014) a020420.

[19] T.M. Mathiisen, K.P. Lehre, N.C. Danbolt, et al., The perivascular astroglial sheath provides a complete covering of the brain microvessels: an electron microscopic 3D reconstruction, Glia 58 (2010) 1094-1103.

[20] T. Molnar, O. Yarishkin, A. Iuso, et al., Store-Operated Calcium Entry in Muller Glia Is Controlled by Synergistic Activation of TRPC and Orai Channels, J Neurosci 36 (2016) 3184-3198.

[21] J. Moss, E. Gebara, E.A. Bushong, et al., Fine processes of Nestin-GFP-positive radial glia-like stem cells in the adult dentate gyrus ensheathe local synapses and vasculature, Proc Natl Acad Sci U S A 113 (2016) E25362545.

[22] I. Patrushev, N. Gavrilov, V. Turlapov, et al., Subcellular location of astrocytic calcium stores favors extrasynaptic neuron-astrocyte communication, Cell Calcium 54 (2013) 343-349.

[23] J. Grosche, V. Matyash, T. Moller, et al., Microdomains for neuron-glia interaction: parallel fiber signaling to Bergmann glial cells, Nat Neurosci 2 (1999) 139-143.

[24] J. Grosche, H. Kettenmann, A. Reichenbach, Bergmann glial cells form distinct morphological structures to interact with cerebellar neurons, J Neurosci Res 68 (2002) 138-149.

[25] F.H. Sterky, S. Lee, R. Wibom, et al., Impaired mitochondrial transport and Parkin-independent degeneration of respiratory chain-deficient dopamine neurons in vivo, Proc Natl Acad Sci U S A 108 (2011) 12937-12942.

[26] A. Agarwal, P.H. Wu, E.G. Hughes, et al., Transient Opening of the Mitochondrial Permeability Transition Pore Induces Microdomain Calcium Transients in Astrocyte Processes, Neuron 93 (2017) 587-605 e587.

[27] L. Ben Haim, D.H. Rowitch, Functional diversity of astrocytes in neural circuit regulation, Nat Rev Neurosci 18 (2017) 31-41.

[28] T.L. Schwarz, Mitochondrial trafficking in neurons, Cold Spring Harb Perspect Biol 5 (2013).

[29] Z.H. Sheng, The Interplay of Axonal Energy Homeostasis and Mitochondrial Trafficking and Anchoring, Trends Cell Biol (2017).

[30] S. Zuchner, I.V. Mersiyanova, M. Muglia, et al., Mutations in the mitochondrial GTPase mitofusin 2 cause Charcot-Marie-Tooth neuropathy type 2A, Nat Genet 36 (2004) 449-451.

[31] C. Alexander, M. Votruba, U.E. Pesch, et al., OPA1, encoding a dynamin-related GTPase, is mutated in autosomal dominant optic atrophy linked to chromosome 3q28, Nat Genet 26 (2000) 211-215.

[32] C. Delettre, G. Lenaers, J.M. Griffoin, et al., Nuclear gene OPA1, encoding a mitochondrial dynamin-related protein, is mutated in dominant optic atrophy, Nat Genet 26 (2000) 207-210.

[33] H.R. Waterham, J. Koster, C.W. van Roermund, et al., A lethal defect of mitochondrial and peroxisomal fission, N Engl J Med 356 (2007) 1736-1741.

[34] M. Liesa, M. Palacin, A. Zorzano, Mitochondrial dynamics in mammalian health and disease, Physiol Rev 89 (2009) 799-845.

[35] S.A. Detmer, D.C. Chan, Functions and dysfunctions of mitochondrial dynamics, Nat Rev Mol Cell Biol 8 (2007) 870-879.

[36] T. MacVicar, T. Langer, OPA1 processing in cell death and disease - the long and short of it, J Cell Sci 129 (2016) 2297-2306.

[37] C.R. Chang, C. Blackstone, Dynamic regulation of mitochondrial fission through modification of the dynaminrelated protein Drp1, Ann N Y Acad Sci 1201 (2010) 34-39.

[38] J.R. Friedman, L.L. Lackner, M. West, et al., ER tubules mark sites of mitochondrial division, Science 334 (2011) 358-362.

[39] N.J. Larsen, G. Ambrosi, S.J. Mullett, et al., DJ-1 knock-down impairs astrocyte mitochondrial function, Neuroscience 196 (2011) 251-264.

[40] T.J. Collins, M.D. Bootman, Mitochondria are morphologically heterogeneous within cells, J Exp Biol 206 (2003) 1993-2000.

[41] T. Liu, C.C. Xue, Y.L. Shi, et al., Overexpression of mitofusin 2 inhibits reactive astrogliosis proliferation in vitro, Neurosci Lett 579 (2014) 24-29. 
[42] J.L. Zamanian, L. Xu, L.C. Foo, et al., Genomic analysis of reactive astrogliosis, J Neurosci 32 (2012) 6391-6410.

[43] J.G. Jackson, J.C. O'Donnell, H. Takano, et al., Neuronal activity and glutamate uptake decrease mitochondrial mobility in astrocytes and position mitochondria near glutamate transporters, J Neurosci 34 (2014) 1613-1624.

[44] T.L. Stephen, N.F. Higgs, D.F. Sheehan, et al., Miro1 Regulates Activity-Driven Positioning of Mitochondria within Astrocytic Processes Apposed to Synapses to Regulate Intracellular Calcium Signaling, J Neurosci 35 (2015) 15996-16011.

[45] A.F. Macaskill, J.E. Rinholm, A.E. Twelvetrees, et al., Miro1 is a calcium sensor for glutamate receptor-dependent localization of mitochondria at synapses, Neuron 61 (2009) 541-555.

[46] D.A. Rusakov, Disentangling calcium-driven astrocyte physiology, Nat Rev Neurosci 16 (2015) 226-233.

[47] A. Volterra, N. Liaudet, I. Savtchouk, Astrocyte $\mathrm{Ca}(2)(+)$ signalling: an unexpected complexity, Nat Rev Neurosci 15 (2014) 327-335.

[48] X. Wang, N. Lou, Q. Xu, et al., Astrocytic Ca2+ signaling evoked by sensory stimulation in vivo, Nat Neurosci 9 (2006) 816-823.

[49] A. Nimmerjahn, E.A. Mukamel, M.J. Schnitzer, Motor behavior activates Bergmann glial networks, Neuron 62 (2009) 400-412.

[50] N. Bazargani, D. Attwell, Astrocyte calcium signaling: the third wave, Nat Neurosci 19 (2016) 182-189.

[51] E. Shigetomi, S. Patel, B.S. Khakh, Probing the Complexities of Astrocyte Calcium Signaling, Trends Cell Biol 26 (2016) 300-312.

[52] E. Shigetomi, X. Tong, K.Y. Kwan, et al., TRPA1 channels regulate astrocyte resting calcium and inhibitory synapse efficacy through GAT-3, Nat Neurosci 15 (2011) 70-80.

[53] M.A. Di Castro, J. Chuquet, N. Liaudet, et al., Local Ca2+ detection and modulation of synaptic release by astrocytes, Nat Neurosci 14 (2011) 1276-1284.

[54] R. Srinivasan, B.S. Huang, S. Venugopal, et al., Ca(2+) signaling in astrocytes from Ip3r2(-/-) mice in brain slices and during startle responses in vivo, Nat Neurosci 18 (2015) 708-717.

[55] K. Kanemaru, H. Sekiya, M. Xu, et al., In vivo visualization of subtle, transient, and local activity of astrocytes using an ultrasensitive $\mathrm{Ca}(2+)$ indicator, Cell Rep 8 (2014) 311-318.

[56] W.J. Nett, S.H. Oloff, K.D. McCarthy, Hippocampal astrocytes in situ exhibit calcium oscillations that occur independent of neuronal activity, J Neurophysiol 87 (2002) 528-537.

[57] R.L. Rungta, L.P. Bernier, L. Dissing-Olesen, et al., Ca2+ transients in astrocyte fine processes occur via Ca2+ influx in the adult mouse hippocampus, Glia 64 (2016) 2093-2103.

[58] P. Bernardi, S. von Stockum, The permeability transition pore as a $\mathrm{Ca}(2+)$ release channel: new answers to an old question, Cell Calcium 52 (2012) 22-27.

[59] H. Qi, L. Li, J. Shuai, Optimal microdomain crosstalk between endoplasmic reticulum and mitochondria for Ca2+ oscillations, Sci Rep 5 (2015) 7984.

[60] A.A. Rowland, G.K. Voeltz, Endoplasmic reticulum-mitochondria contacts: function of the junction, Nat Rev Mol Cell Biol 13 (2012) 607-625.

[61] D. De Stefani, R. Rizzuto, T. Pozzan, Enjoy the Trip: Calcium in Mitochondria Back and Forth, Annu Rev Biochem 85 (2016) 161-192.

[62] H. Li, X. Wang, N. Zhang, et al., Imaging of mitochondrial Ca2+ dynamics in astrocytes using cell-specific mitochondria-targeted GCaMP5G/6s: mitochondrial $\mathrm{Ca} 2+$ uptake and cytosolic $\mathrm{Ca} 2+$ availability via the endoplasmic reticulum store, Cell Calcium 56 (2014) 457-466.

[63] J. Parnis, V. Montana, I. Delgado-Martinez, et al., Mitochondrial exchanger NCLX plays a major role in the intracellular Ca2+ signaling, gliotransmission, and proliferation of astrocytes, J Neurosci 33 (2013) 7206-7219.

[64] J. Kolikova, R. Afzalov, A. Giniatullina, et al., Calcium-dependent trapping of mitochondria near plasma membrane in stimulated astrocytes, Brain Cell Biol 35 (2006) 75-86.

[65] T.T. Nguyen, S.S. Oh, D. Weaver, et al., Loss of Miro1-directed mitochondrial movement results in a novel murine model for neuron disease, Proc Natl Acad Sci U S A 111 (2014) E3631-3640.

[66] J.G. Jackson, M.B. Robinson, Reciprocal Regulation of Mitochondrial Dynamics and Calcium Signaling in Astrocyte Processes, J Neurosci 35 (2015) 15199-15213.

[67] T. Misgeld, M. Kerschensteiner, F.M. Bareyre, et al., Imaging axonal transport of mitochondria in vivo, Nat Methods 4 (2007) 559-561.

[68] S. Lee, K.S. Lee, S. Huh, et al., Polo Kinase Phosphorylates Miro to Control ER-Mitochondria Contact Sites and Mitochondrial Ca(2+) Homeostasis in Neural Stem Cell Development, Dev Cell 37 (2016) 174-189.

[69] W.P. Ge, A. Miyawaki, F.H. Gage, et al., Local generation of glia is a major astrocyte source in postnatal cortex, Nature 484 (2012) 376-380.

[70] J. Jacobson, M.R. Duchen, Mitochondrial oxidative stress and cell death in astrocytes--requirement for stored Ca2+ and sustained opening of the permeability transition pore, J Cell Sci 115 (2002) 1175-1188.

[71] V.C. Keil, F. Funke, A. Zeug, et al., Ratiometric high-resolution imaging of JC-1 fluorescence reveals the subcellular heterogeneity of astrocytic mitochondria, Pflugers Arch 462 (2011) 693-708.

[72] S. Deheshi, B. Dabiri, S. Fan, et al., Changes in mitochondrial morphology induced by calcium or rotenone in primary astrocytes occur predominantly through ros-mediated remodeling, J Neurochem 133 (2015) 684-699. 
[73] A.R. Tan, A.Y. Cai, S. Deheshi, et al., Elevated intracellular calcium causes distinct mitochondrial remodelling and calcineurin-dependent fission in astrocytes, Cell Calcium 49 (2011) 108-114.

[74] W. Fu, A. Ruangkittisakul, D. MacTavish, et al., Activity and metabolism-related Ca2+ and mitochondrial dynamics in co-cultured human fetal cortical neurons and astrocytes, Neuroscience 250 (2013) 520-535.

[75] D. Naon, M. Zaninello, M. Giacomello, et al., Critical reappraisal confirms that Mitofusin 2 is an endoplasmic reticulum-mitochondria tether, Proc Natl Acad Sci U S A 113 (2016) 11249-11254.

[76] M.Z. Lin, M.J. Schnitzer, Genetically encoded indicators of neuronal activity, Nat Neurosci 19 (2016) 1142-1153.

[77] R. Srinivasan, T.Y. Lu, H. Chai, et al., New Transgenic Mouse Lines for Selectively Targeting Astrocytes and Studying Calcium Signals in Astrocyte Processes In Situ and In Vivo, Neuron 92 (2016) 1181-1195.

[78] M.E. Hamby, G. Coppola, Y. Ao, et al., Inflammatory mediators alter the astrocyte transcriptome and calcium signaling elicited by multiple g-protein-coupled receptors, J Neurosci 32 (2012) 14489-14510.

[79] U. Ito, Y. Hakamata, E. Kawakami, et al., Degeneration of astrocytic processes and their mitochondria in cerebral cortical regions peripheral to the cortical infarction: heterogeneity of their disintegration is closely associated with disseminated selective neuronal necrosis and maturation of injury, Stroke 40 (2009) 2173-2181.

[80] S. Schmidt, B. Linnartz, S. Mendritzki, et al., Genetic mouse models for Parkinson's disease display severe pathology in glial cell mitochondria, Hum Mol Genet 20 (2011) 1197-1211.

[81] O.J. Castejon, Biopathology of astrocytes in human traumatic and complicated brain injuries. Review and hypothesis, Folia Neuropathol 53 (2015) 173-192.

[82] G. Harish, A. Mahadevan, N. Pruthi, et al., Characterization of traumatic brain injury in human brains reveals distinct cellular and molecular changes in contusion and pericontusion, J Neurochem 134 (2015) 156-172.

[83] I.S. Balan, A.J. Saladino, B. Aarabi, et al., Cellular alterations in human traumatic brain injury: changes in mitochondrial morphology reflect regional levels of injury severity, J Neurotrauma 30 (2013) 367-381.

[84] E. Lezi, R.H. Swerdlow, Mitochondria in neurodegeneration, Adv Exp Med Biol 942 (2012) 269-286.

[85] M.D. Norenberg, K.V. Rao, The mitochondrial permeability transition in neurologic disease, Neurochem Int 50 (2007) 983-997.

[86] M.J. Bird, D.R. Thorburn, A.E. Frazier, Modelling biochemical features of mitochondrial neuropathology, Biochim Biophys Acta 1840 (2014) 1380-1392.

[87] L.D. Osellame, A.A. Rahim, I.P. Hargreaves, et al., Mitochondria and quality control defects in a mouse model of Gaucher disease--links to Parkinson's disease, Cell Metab 17 (2013) 941-953.

[88] P.A. Parone, S. Da Cruz, J.S. Han, et al., Enhancing mitochondrial calcium buffering capacity reduces aggregation of misfolded SOD1 and motor neuron cell death without extending survival in mouse models of inherited amyotrophic lateral sclerosis, J Neurosci 33 (2013) 4657-4671.

[89] P. Cassina, A. Cassina, M. Pehar, et al., Mitochondrial dysfunction in SOD1G93A-bearing astrocytes promotes motor neuron degeneration: prevention by mitochondrial-targeted antioxidants, J Neurosci 28 (2008) 41154122.

[90] A. San Martin, T. Sotelo-Hitschfeld, R. Lerchundi, et al., Single-cell imaging tools for brain energy metabolism: a review, Neurophotonics 1 (2014) 011004.

[91] G.C. Brown, J.P. Bolanos, S.J. Heales, et al., Nitric oxide produced by activated astrocytes rapidly and reversibly inhibits cellular respiration, Neurosci Lett 193 (1995) 201-204.

[92] M.J. Barsoum, H. Yuan, A.A. Gerencser, et al., Nitric oxide-induced mitochondrial fission is regulated by dynamin-related GTPases in neurons, EMBO J 25 (2006) 3900-3911.

[93] B. Westermann, Mitochondrial fusion and fission in cell life and death, Nat Rev Mol Cell Biol 11 (2010) $872-884$.

[94] M. Nagai, D.B. Re, T. Nagata, et al., Astrocytes expressing ALS-linked mutated SOD1 release factors selectively toxic to motor neurons, Nat Neurosci 10 (2007) 615-622.

[95] K. Yamanaka, S.J. Chun, S. Boillee, et al., Astrocytes as determinants of disease progression in inherited amyotrophic lateral sclerosis, Nat Neurosci 11 (2008) 251-253.

[96] S.T. Papadeas, S.E. Kraig, C. O'Banion, et al., Astrocytes carrying the superoxide dismutase 1 (SOD1G93A) mutation induce wild-type motor neuron degeneration in vivo, Proc Natl Acad Sci U S A 108 (2011) 1780317808.

[97] K. Hayakawa, E. Esposito, X. Wang, et al., Transfer of mitochondria from astrocytes to neurons after stroke, Nature 535 (2016) 551-555.

[98] M.V. Berridge, M.J. McConnell, C. Grasso, et al., Horizontal transfer of mitochondria between mammalian cells: beyond co-culture approaches, Curr Opin Genet Dev 38 (2016) 75-82.

[99] C.H. Davis, K.Y. Kim, E.A. Bushong, et al., Transcellular degradation of axonal mitochondria, Proc Natl Acad Sci U S A 111 (2014) 9633-9638.

[100] M.V. Berridge, R.T. Schneider, M.J. McConnell, Mitochondrial Transfer from Astrocytes to Neurons following Ischemic Insult: Guilt by Association?, Cell Metab 24 (2016) 376-378.

[101] S.A. Liddelow, K.A. Guttenplan, L.E. Clarke, et al., Neurotoxic reactive astrocytes are induced by activated microglia, Nature 541 (2017) 481-487.

[102] M.A. Anderson, J.E. Burda, Y. Ren, et al., Astrocyte scar formation aids central nervous system axon regeneration, Nature 532 (2016) 195-200. 


\section{Figure legends}

\section{Figure 1: Mitochondrial network architecture in astroglial cells in vivo}

(A) Schematic illustration of a viral-based approach to express mitochondrial-targeted GFP (mtGFP) in astrocytes. On the right, representative confocal pictures show the elaborated mitochondrial network of reconstructed mtGFP-expressing astrocytes across different layers of the neocortex. Bars $10 \mu \mathrm{m}$. (B) Utilization of an inducible reporter mouse to express mito-YFP (mtYFP) in the ROSA26 locus[25] specifically in astrocytes. Right images depict representative high-resolution acquisitions of mtYFP-expressing astroglia in different brain regions (see insets) including cortex, cerebellum (Bergmann glia), hippocampus and striatum. The yellow arrowheads point to astroglia cell bodies. Bars $10 \mu \mathrm{m}$.

\section{Figure 2: Assessment of mitochondrial motility in astrocytes in vitro and ex vivo}

(A) Time-lapse imaging of astrocytes in culture expressing mtGFP. Images show individual mitochondria tracked over the course of 1 minute and their relative classification according to their motility[16]. (B) Color-coded tracks of selected mitochondria are shown. Scale bar: $5 \mu \mathrm{m}$. (C) Time-lapse sequence of an mtGFP-expressing astrocyte ex vivo following viral transduction in vivo. Images show the distribution of astrocytic mitochondria during 30 minutes of imaging. Bar $20 \mu \mathrm{m}$. (D) Superimposition of the frames shown in C. Mitochondria which had moved between frames appear in single colors. Bar $20 \mu \mathrm{m}$. (E and F) High magnifications of the two areas boxed in D depicting mitochondria stable over time $(\mathbf{E})$ compared to a slowly-moving mitochondrion $(\mathbf{F})$. Bars $10 \mu \mathrm{m}$. (G) Assessment of the overall mitochondrial displacement in four individual astrocytes expressing mtGFP and examined every 10 minutes during 2 hours of imaging. 
Figure 1

A

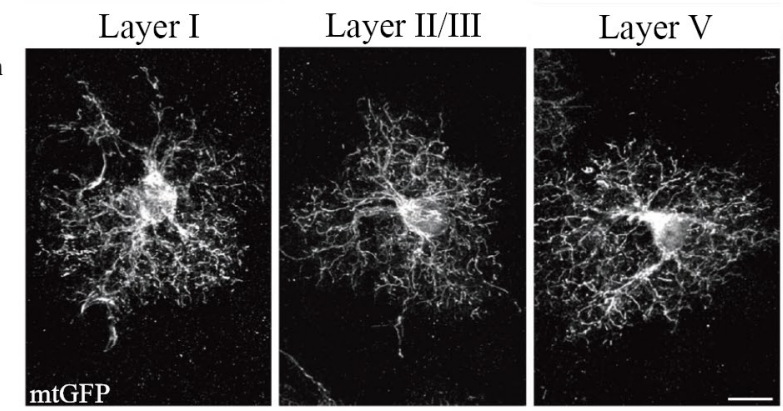

B

Viral-based expression

of mitochondrial

targeted indicators

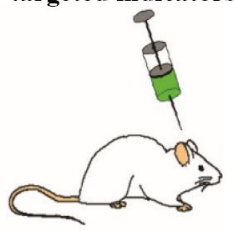

mtGFP
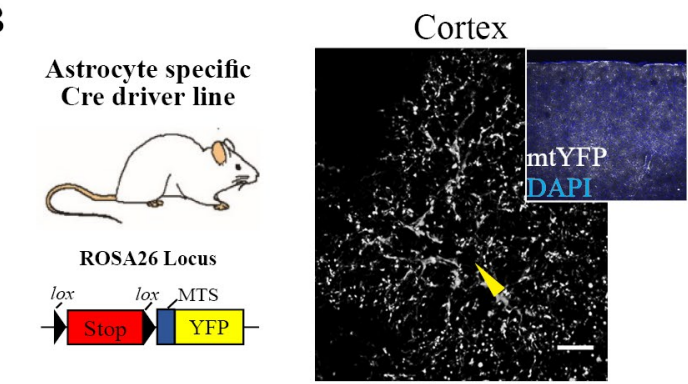

Cerebellum

Hippocampus
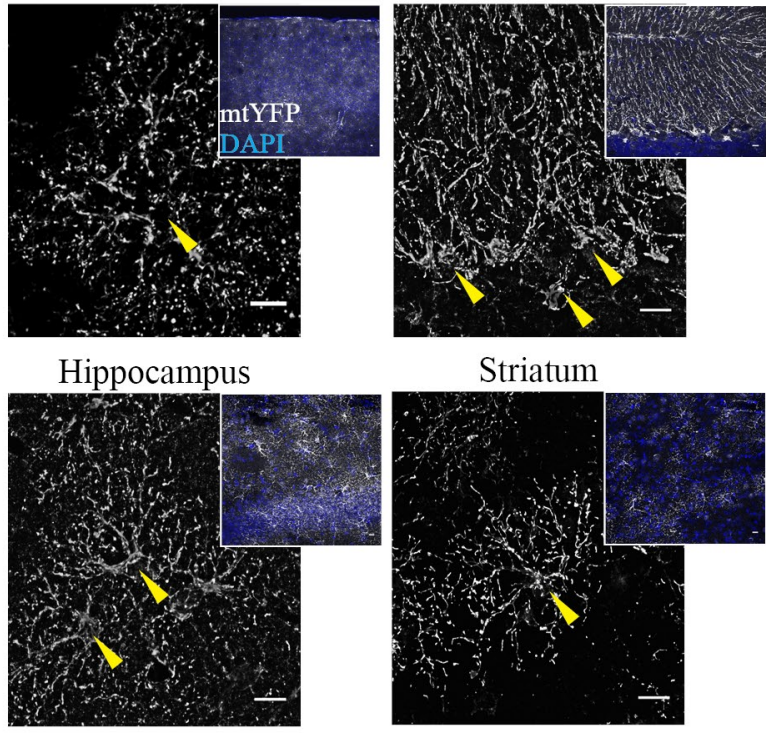

Striatum

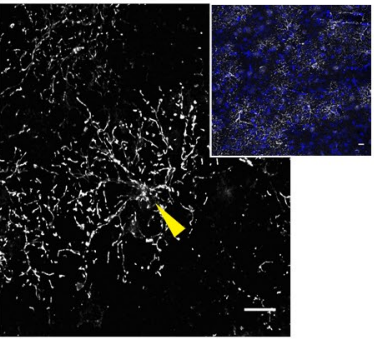


Figure 2

A
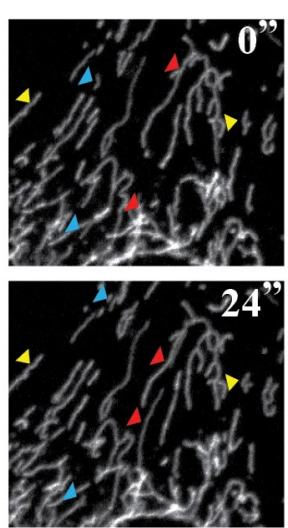

C

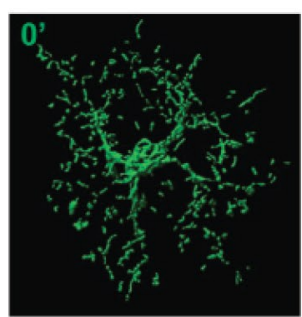

D

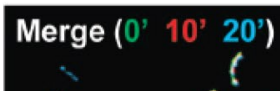

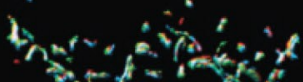

-1 Na $=$

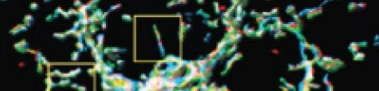

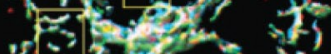

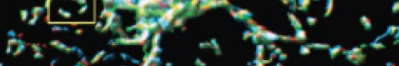

"रो.

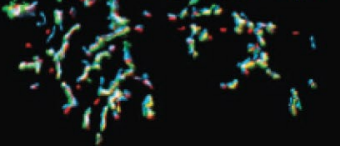

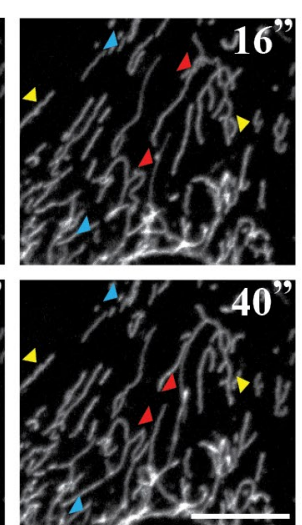

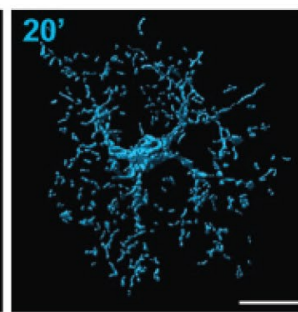

$\mathbf{F}$
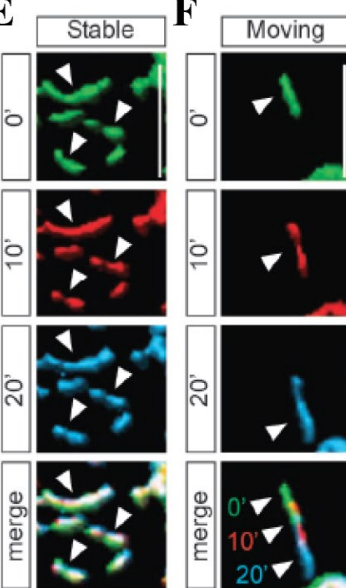

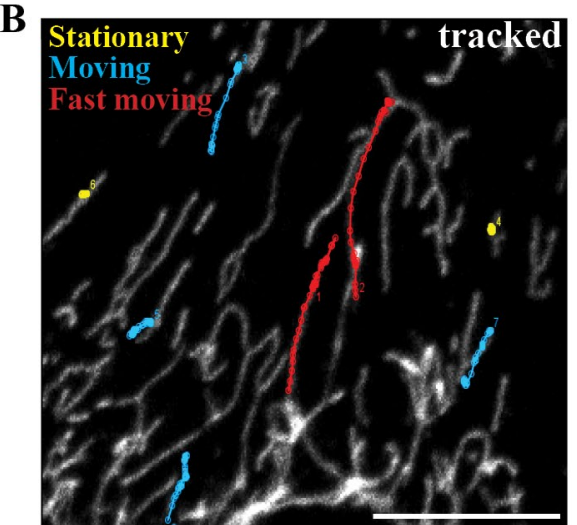

G

Mitochondrial displacement

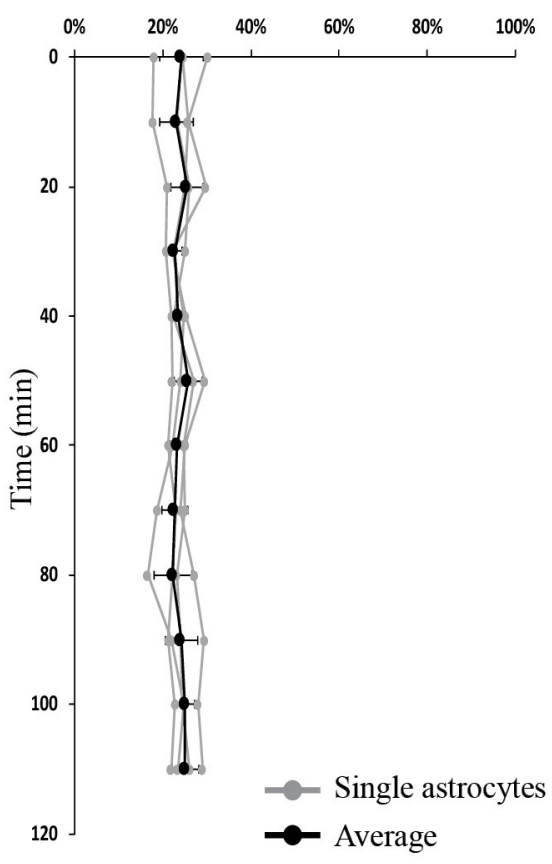




\section{${ }^{\star}$ Conflict of Interest}

To view the full contents of this document, you need a later version of the PDF viewer. You can upgrade to the latest version of Adobe Reader from www.adobe.com/products/acrobat/readstep2.html

For further support, go to www.adobe.com/support/products/acrreader.html 